\title{
Review of Autoimmune Hepatitis: Diagnosis and Treatment
}

\author{
Minkoulou Danielle, Yongfeng Yang \\ Liver Disease Department, The Second Hospital of Nanjing, South East University, Nanjing, China \\ Email: yyf1997@163.com
}

How to cite this paper: Danielle, M. and Yang, Y.F. (2018) Review of Autoimmune Hepatitis: Diagnosis and Treatment. Open Journal of Gastroenterology, 8, 57-66. https://doi.org/10.4236/ojgas.2018.83006

Received: February 12, 2018

Accepted: March 12, 2018

Published: March 15, 2018

Copyright (C) 2018 by authors and Scientific Research Publishing Inc. This work is licensed under the Creative Commons Attribution International License (CC BY 4.0).

http://creativecommons.org/licenses/by/4.0/

\begin{abstract}
Background: Autoimmune hepatitis (AIH) is a hepatocyte injury characterized by a dis-regulated immune system, inflammation of the liver with interface hepatitis, serum antibodies and elevated gamma-globulins. Currently, it exists 2 mainly types on autoimmune hepatitis, based on the presentation of their autoantibodies: type 1 and 2. Type 1 is the one of interest in this review. Autoimmune hepatitis type 1 is an indolent inflammatory disease mediated by abnormal autoimmune reaction, commonly seeing in women (4:1), with no preference of age, however typically present in 4th and 6th decade's individuals, and a good response to immunosuppressive therapy. Autoimmune hepatitis is known to present not specific symptoms which can go from inexistent to fulminant or exacerbate presentation. The incidence of autoimmune hepatitis in western country is $1 / 5000-1 / 10,000$. Although the detection rate of AIH increasing, especially in China, autoimmune hepatitis diagnosis and treatment remain challenging. Most AIH case do not present pathognomonic biomarkers, however the diagnostic has to considerate other features, which had been codified into validated diagnosis scoring systems. Regarding the treatment, based on prior literature autoimmune hepatitis is mostly sensitive to prednisone or azathioprine, however the use of other immunosuppressive agent or event their combination had offer distinct advantages. Autoimmune hepatitis presents a great life threatening disorder especially because of the delay in the early stage on the disease, the not specific presentations, and the large overlap syndrome associate with it. A deep knowledge in its identification, specific diagnostic criteria, and its therapeutic strategy is much needed. Conclusion: Autoimmune hepatitis is a systemic disease that is difficult to recognize because of its variable clinical presentation and histological features that are not strictly specific. Several new autoantibodies as well as recently simplified diagnostic criteria may allow the primary care physician to progress in the diagnostic process. This is all the more important as undiagnosed and therefore
\end{abstract}


untreated autoimmune hepatitis has a poor prognosis and immunosuppressive treatment leads to remission in a large majority of cases.

\section{Keywords}

Autoimmune Hepatitis, Diagnosis, Immunosoppressive Therapy

\section{Introduction}

Autoimmune hepatitis firstly described by Waldenström and Kunkel in the 1950s, inherited a variety of nomenclature, including chronic active hepatitis, cirrhosis of young women and plasma cell hepatitis [1]. In 1959, Mc Kay again used the term of lupoid hepatitis to characterize this disease, which shares clinical and biological similarities with systemic lupus erythematosus, but with an initial and predominant liver injury. Autoimmune hepatitis is a chronic inflammatory disease that can occur at any age, in both sexes of all ethnic origins, but which more readily affects women (F:M ratio of 3.6:1). Its prevalence is estimated at $10-17 / 100,000$ in Europe [2]. Along with primary biliary cirrhosis (PBC) and primary sclerosing cholangitis (PSC), AIH is an autoimmune liver disease. It is essential to know how to differentiate AIH from other chronic hepatitis because a treatment immunosuppressant is often effective. In the absence of treatment, it has a relatively poor prognosis with a survival that hardly exceeds $10 \%$ at ten years [3] [4] [5].

The pathogenesis of AIH is not completely elucidated, but the hypothesis implies a genetic predisposition including in particular the genes of the major histocompatibility complex (HLA). For example, in North America and Europe, the HLA-DR3 and HLA-DR4 alleles would be a susceptibility factor. At the environmental level, an immune response to an external pathogen, drug or toxin trigger could erroneously target structurally similar internal components (mimicry, molecular). This breakdown of immune tolerance would then lead to an innate and acquired immune response that would self-sustain. In case of dysfunction of regulatory $\mathrm{T}$ cells, which inter alia prevent autoimmunity, the mechanism of destruction of hepatocytes may persist. The latter is mediated in AIH by cytotoxic $\mathrm{T}$ lymphocytes $(\mathrm{CD} 8+\mathrm{T})$, cytokines secreted by Th1 lymphocytes, monocytes, macrophages, NK lymphocytes and complement activation [6] [7]. The autoantibodies sought for AIH diagnosis does not seem to have a pathogenic role.

Autoimmune hepatitis is characterized by three elements: 1) a particular lesion, the interface hepatitis; 2) the absence of other causes of this lesion (viral hepatitis, drug hepatitis, Wilson's disease or alpha-1 antitrypsin deficiency and 3) signs of autoimmunity (autoantibodies or other autoimmune disorders). A complex international score was established by the International Autoimmune Hepatitis Group (IAHG) for a mainly academic purpose [8]. Recently a new approach, of simplified criteria, commonly use in clinical practice [9] [10]. The 
study presented the new simplified scoring methods in Table 1 . The excellent specificity of these simplified criteria has been confirmed in an even more recent study; however, this method is wildly used in clinical practice, its sensitivity still remain lower, so it should always make use of the original IAIHG scoring method [11].

\section{Diagnosis}

\subsection{Symptoms}

Concerning the clinical presentation nearly one-third of AIH patients are asymptomatic, which may contribute to late recognition of the disease [12]. The onset is often insidious, with symptoms and signs nonspecific or related to liver damage (abdominal pain, jaundice, hepatomegaly, splenomegaly). Frequently it's an elevation chronic ( $>3$ to 6 months) transaminases (AST, ALT) that holds the doctor's attention. However more rarely, AIH can manifest as fulminant hepatitis (jaundice, transaminases $>1000 \mathrm{IU} / \mathrm{l}$ ) [13].

\subsection{Atypical Form of AIH}

$\mathrm{AIH}$ is considered as a disorder with atypical clinical and histological features, based on the literature Caucasian woman has no exclusivity for autoimmune hepatitis, although she has long been considered the typical terrain. In addition, histological finding is characterized by a lesion in which interface hepatitis is not always present, or not the most being changes in the hepatocytes age had always been associated to $\mathrm{AIH}$, specially over 40th and wish correspond to the peak if incidence.

Table 1. AIH simplified criteria.

\begin{tabular}{ccc}
\hline Features/parameter & Discriminator & score \\
\hline ANA or SMA+ & $\geq 1: 40$ & $+1^{*}$ \\
ANA or SMA+ & $\geq 1: 80$ & +2 \\
or LKM+ & $\geq 1: 40$ & +2 \\
Or SLA/LP+ & Any titer & +2 \\
IgG or gamma-globulin level & $>$ upper limit of normal & +1 \\
Liver histology & $>1.1$ upper limit & +2 \\
(evidence of hepatitis ) & Compatible with AIH & +1 \\
& Typical of AIH & +2 \\
Absence of hepatitis & Atypical & 0 \\
& No & 0
\end{tabular}

Definite Autoimmune hepatitis: $\geq 7$; probable autoimmune hepatitis $\geq 6$ [21]. 
$\mathrm{AIH}$ is said to also be influenced by race as black subjects present generally more serious manifestations, and this especially male. Cirrhosis is more common at the time of diagnosis; fibrosis progression is more rapid, and the terminal stage of cirrhosis is more frequently reached [14] [15], Asian AIH patients, on the other hand, demonstrated a higher rate of mortality and thus displayed the worst survival curve [16].

Men are clearly less exposed to autoimmune hepatitis but they are not spared [17] [18] [19].

It has been individualized a form where the initial lesions sit in the centrolobular region (necrosis with mononucleated inflammatory infiltrate, without steatohepatitis), while the interface hepatitis is absent or minimal. Lesions around the periportal region appear secondarily. This form responds well to immunosuppressive therapy [20]. Differential diagnosis of this form with an acute immune allergic hepatitis is difficult.

\subsection{Overall Syndrome}

Autoimmune hepatitis has multiple overlap syndromes differential diagnosis of autoimmune hepatitis, differential diagnoses of autoimmune hepatitis: diagnosis Clinical or laboratory elements suggestive of diagnosis Primary sclerosing cholangitis, Primary biliary cirrhosis, Acute viral hepatitis (History of exposure, origin of patients A, B, C, D, E, CMV, Serology, possibly EBV viremia, HSV, Hepatitis) Drug history or toxic drug, suspected intoxication Potential re-exposure Alcoholic hepatitis Anamnesis of alcohol abuse, nonalcoholic steatohepatitis (NASH): Nonalcoholic steatohepatitis, Hemochromatosis, Alpha-1-antitrypsin deficiency, phenotypic variant Wilson's disease.

\subsection{Laboratory Examinations}

Laboratory tests often show a significant increase in transaminases (AST, ALT). Bilirubin and alkaline phosphatase are usually normal or slightly increased.14 Otherwise, an overlap syndrome with PBC or PSC should be sought. An elevation of gamma globulins was found in $90 \%$ of cases, with IgG at 1.5 - 3 times the norm in $85 \%$ of patients with HAI. Gamma-globulin elevation is found in all chronic liver diseases, but most often at lower rates only in the AIH. The mechanism involves a reduction in clearance by Kupffer cells of antigens delivered by the portal venous system, resulting in increased exposure in the systemic circulation and antibody production sites. As a result, the increase in IgG is rather a consequence of liver disease rather than the reverse.

\subsection{Immunological Markers}

The immunological markers make it possible both to specify the diagnosis and to evaluate the prognosis to a certain extent. Moreover, in $10 \%$ to $25 \%$ of cases, patients with AIH have low or undetectable levels of autoantibodies.

AIH Type 1 (classical form) accounts for about 75\% of HAI cases. The anti- 
nuclear factor, found in more than $90 \%$ of cases, is not very specific. The presence of smooth anti-muscle antibodies can sometimes differentiate AIH from systemic lupus erythematosus. Anti-actin antibodies are more specific and more sensitive than smooth anti-muscles. Their presence is associated with an unfavorable prognosis. Anti-SLA or anti-SLA/LP is the most specific marker of the disease. It is found in $10 \%$ to $30 \%$ of adults with AIH type 1. p-ANCA (perinuclear anti-neutrophil cytoplasmic antibodies) can also be detected, but their research is of limited value for diagnosis, as is the search for anti-mitochondria (usually found in PBC, but also in 5\% to $20 \%$ of AIH type 1). Native anti-DNA, a relatively specific marker of systemic lupus erythematosus, can also be seen in AIH type 1.

\subsection{Diagnostic Score}

A diagnostic score was published by IAIHG (International Autoimmune Hepatitis Group) in 1993 [22] [23] and revised in 1999 [8]. A new promising score from retrospective studies is currently available [9] [10]. Although it still needs to be validated prospectively, it has advantage, thanks to its simplicity, to be more adapted to the screening performed by the practitioner (Table 1). Its sensitivity would be greater than $85 \%$ and its specificity of $95 \%$, which makes it especially a good tool of exclusion. In clinical practice, in some uncertain cases, the response to a short-term corticosteroids may be helpful in the development of the diagnosis [2].

\subsection{Liver Biopsy}

The histological aspect of AIH is that of chronic hepatitis with some characteristic signs that can help the diagnosis, but are not completely specific. It is most often the combination of portal and periportal inflammation (typically predominantly plasma cell) associated interface hepatitis, rosette formation, emperipolesis. There is usually no damage to the bile ducts. The latter would rather suggest a syndrome of overlap with the PBC or the PSC.

Compound of portal and periportal infiltrates lymphocytes, macrophages and plasma cells associated with an interface hepatitis, typical of autoimmune hepatitis.

\section{Treatment}

The prognosis of untreated AIH is relatively poor. In contrast, immunosuppressive therapy is effective in $65 \%$ to $80 \%$ of cases [7]. If the indication of the treatment of all children as well as patients with AIH with moderate or severe inflammation (AST $>5 \mathrm{x}$ standard, gamma-globulins $>2 \mathrm{x}$ standard, liver biopsy showing confluent necrosis) is clear, whether to treat asymptomatic adults or not, with only mild sign of hepatitis as an interface, is less obvious and should be discussed case by case. Patients with cirrhosis inactive do not need to be treated. An untreated patient should receive follow-up every three to six months and a 
new histological evaluation within two years [2].

\section{Corticosteroide and Immunosoppressive}

The recommended treatment is to combine average doses of corticosteroids (prednisone or prednisolone $30 \mathrm{mg} /$ day) with azathioprine $(1 \mathrm{mg} / \mathrm{kg} /$ day). These doses can be doubled in case of resistance to treatment. Corticosteroids can then be steadily stopped over approximately two months, depending on the course. Autoantibody levels should not be used to evaluate the response to treatment. In non-cirrhotic patients and with significant corticosteroid-related adverse events, budesonide $9 \mathrm{mg}$ /day Be an alternative [24] [25]. Indeed, when administered orally, budesonide has an important first-pass effect (90\%) and has fewer systemic adverse effects than standard systemic corticosteroids. If AIH is still a problem or azathioprine is not tolerated, other immunosuppressive may be offered. However, no randomized study exists regarding these alternatives. Mycophenolate mofetil $2 \mathrm{~g}$ /day is an interesting option, as is cyclosporine $\mathrm{A}$, tacrolimus or cyclophosphamide. TNF antagonists are controversial. Finally, some patients have been successfully treated with methotrexate or rituximab. Ursodeoxycholic acid does not benefit, contrary to what is observed in the PBC [2].

\section{Follow-Up and Duration of Treatment}

The monitoring, conducted jointly by immunologists and hepatologists, is based on the clinical and biological response using transaminases and IgG. A biological and histological remission is rarely reached before twelve months. Therefore, the duration of treatment is at least two years, clinician recommend liver biopsy before thinking of stopping the treatment, however liver biopsy can still present interface hepatitis, even after the normalization of transaminases and IgG, which requires further treatment. Knowing that the Most patients re-offend in the months following a therapeutic interruption, cases should be evaluated in case the potential risks/benefits to continue low-dose treatment, which is often enough to control the disease. In pediatric cases, it is reasonable to continue long-term therapy [2].

\section{Associated Treatments}

Vaccination against hepatitis A and B is recommended before initiating immunosuppressive therapy. Because of corticosteroids, ophthalmologic monitoring is indicated, as well as prophylaxis of corticosteroid-induced osteoporosis with calcium, vitamin D and possibly bisphosphonates [3].

\section{Transplantation}

Patients presenting with fulminant hepatitis, threatening survival, should be referred to a specialized center for potential liver transplantation, which may be indicated according to the same criteria as for other hepatopathies. Surprisingly, a recurrence rate of $\mathrm{AIH}$ is observed in $10 \%$ to $35 \%$ of transplant recipients, de- 
spite the immunosuppressive treatment. AIH in transplant patients have also been described. 5, 6 However, AIH remains an excellent indication for transplantation with survival rates of $90 \%$ at five years and $75 \%$ at ten years of age [26].

\section{Relative Indications: Need Not Be Treated?}

In the 2014 Beijing liver disease, infectious disease conference, University professor Wang Guiqiang director of diseases and liver disease center. Professor Wang Guiqiang introduced the status of diagnosis and treatment of autoimmune hepatitis in China and shared the experience of improving patient compliance.

In the past 15 years, there has been little change in the international guidelines for the treatment of autoimmune hepatitis. But we have done some experiments with China's national conditions and our clinical experience, and here we share it with you. First, the indications in the foreign guidelines include absolute and relative indications. Absolute indication is that transaminase is more than 10 times higher than normal, or transaminase is more than 5 times normal, immunoglobulin is more than two times, or pathology has obvious lobular necrosis, bridging necrosis and other significant lesions. All of them need immediate treatment. Other cases are relative indications.

\section{Treatment and Prognosis}

\subsection{Definition of the Response Criteria}

Although the reference remains the disappearance of lobular and portal necrotic and inflammatory lesions, a consensus seems to be acquired around a purely biochemical definition. Although a certain degree of heterogeneity persists, an increasingly broad consensus is emerging around the following definitions:

- Complete (Biochemical) Remission: Transaminases Within Persistent Normal Values.

- Partial remission: Transaminases $<2$ times the upper limit of normal values (x ULN).

- Relapse: Increase in transaminases has a value greater than the normal values after their normalization.

- Failure of treatment: Stability or rise of transaminases.

These definitions are based on the very good relationship between lesions of lobular and periportal necrosis or inflammation and transaminases on the one hand, [22] [23], the overall evolution of the disease, on the other hand [24] [25] [26]. These definitions therefore tend to replace the histological criteria of response, but also the biochemical criteria used in the Mayo Clinic studies. (AST $<2 \mathrm{x}$ ULN for remission and AST $>3 \mathrm{x}$ ULN for relapse).

\subsection{Complications}

Like portal hypertension and cellular insufficiency, hepatocellular carcinoma is a 
potential complication of HAI in the cirrhosis stage. Therefore, a liver ultrasound screening is recommended every six months [2].

\section{Conclusion}

Autoimmune hepatitis is a rare disease; the etiopathogenesis is not yet elucidated. Although treatment has changed little in recent years, recent diagnostic advances make it easier to screen for this disease. Future randomized studies with new immunosuppressive and biologic therapies should provide better management of patients, especially in cases of resistance, contraindication or intolerance to corticosteroids or azathioprine. It is therefore recommended to refer these patients to a center specialized for joint management by immunologists and hepatologists.

\section{Practical Implications}

Autoimmune hepatitis is more prevalent among young women, but can affect all ethnicities, all ages, and both sexes

The signs and symptoms are not very specific and a third of the patients are asymptomatic. Unfortunately, this contributes to a late discovery of the disease, sometimes at the stage of cirrhosis

- There are new simplified diagnostic scores that may be useful to the primary care physician

- Autoimmune hepatitis may be associated with other systemic diseases that should also be investigated, such as colitis ulcerative, autoimmune thyroiditis, celiac disease and rheumatoid arthritis

- Immunosuppressive therapies are often effective and frequently prevent progression to cirrhosis

\section{References}

[1] Reuben, A. (2003) A Sheep in Wolf's Clothing. Hepatology, 38, 1596-1601. https://doi.org/10.1002/hep.510380643

[2] Gleeson, D. and Heneghan, M.A. (2011) British Society of Gastroenterology (BSG) Guidelines for Management of Autoimmune Hepatitis. Gut, 60, 1611-1629. https://doi.org/10.1136/gut.2010.235259

[3] Manns, M.P., et al. (2010) Diagnosis and Management of Autoimmune Hepatitis. Hepatology, 51, 2193-2213. https://doi.org/10.1002/hep.23584

[4] Chinese Society of Hepatology, C.M.A., C.M.A. Chinese Society of Gastroenterology, and C.M.A. Chinese Society of Infectious Diseases (2016) Chinese Consensus on the Diagnosis and Management of Autoimmune Hepatitis (2015). Zhonghua Gan Zang Bing Za Zhi, 24, 23-35.

[5] Czaja, A.J. (2016) Diagnosis and Management of Autoimmune Hepatitis: Current Status and Future Directions. Gut Liver, 10, 177-203. https://doi.org/10.5009/gnl15352

[6] Vergani, D. and Mieli-Vergani, G. (2008) Aetiopathogenesis of Autoimmune Hepatitis. World Journal of Gastroenterology, 14, 3306-3312. https://doi.org/10.3748/wjg.14.3306 
[7] Longhi, M.S., et al. (2010) Aetiopathogenesis of Autoimmune Hepatitis. Journal of Autoimmunity, 34, 7-14. https://doi.org/10.1016/j.jaut.2009.08.010

[8] Alvarez, F., et al. (1999) International Autoimmune Hepatitis Group Report: Review of Criteria for Diagnosis of Autoimmune Hepatitis. Journal of Hepatology, 31, 929-938. https://doi.org/10.1016/S0168-8278(99)80297-9

[9] Hennes, E.M., et al. (2008) Simplified Criteria for the Diagnosis of Autoimmune Hepatitis. Hepatology, 48, 169-176. https://doi.org/10.1002/hep.22322

[10] Candia, R., et al. (2017) Validation of the Simplified Criteria for the Diagnosis of Autoimmune Hepatitis in Chilean-Hispanic Patients. Annals of Hepatology, 16, 772-779. https://doi.org/10.5604/01.3001.0010.2787

[11] Czaja, A.J. (2008) Performance Parameters of the Diagnostic Scoring Systems for Autoimmune Hepatitis. Hepatology, 48, 1540-1548. https://doi.org/10.1002/hep.22513

[12] Kogan, J., et al. (2002) Prognosis of Symptomatic versus Asymptomatic Autoimmune Hepatitis: A Study of 68 Patients. Journal of Clinical Gastroenterology, 35, 75-81. https://doi.org/10.1097/00004836-200207000-00016

[13] Krawitt, E.L. (2006) Autoimmune Hepatitis. The New England Journal of Medicine, 354, 54-66. https://doi.org/10.1056/NEJMra050408

[14] Lim, K.N., et al. (2001) Autoimmune Hepatitis in African Americans: Presenting Features and Response to Therapy. The American Journal of Gastroenterology, 96, 3390-3394. https://doi.org/10.1111/j.1572-0241.2001.05272.x

[15] Verma, S., Torbenson, M. and Thuluvath, P.J. (2007) The Impact of Ethnicity on the Natural History of Autoimmune Hepatitis. Hepatology, 46, 1828-1835. https://doi.org/10.1002/hep.21884

[16] Liberal, R. and Vergani, D. (2012) Effect of Ethnicity on the Clinical Presentation and Outcome of Autoimmune Hepatitis. Expert Review of Gastroenterology \& $\mathrm{He}-$ patology, 6, 267-269. https://doi.org/10.1586/egh.12.17

[17] Heneghan, M.A. (2002) Autoimmune Hepatitis in Men: Examining the Gender Gap. The American Journal of Gastroenterology, 97, 1870-1872. https://doi.org/10.1111/j.1572-0241.2002.05897.x

[18] Al-Chalabi, T., et al. (2008) Impact of Gender on the Long-Term Outcome and Survival of Patients with Autoimmune Hepatitis. Journal of Hepatology, 48, 140-147. https://doi.org/10.1016/j.jhep.2007.08.013

[19] Bittencourt, P.L., et al. (2008) Frequency of Concurrent Autoimmune Disorders in Patients with Autoimmune Hepatitis: Effect of Age, Gender, and Genetic Background. Journal of Clinical Gastroenterology, 42, 300-305. https://doi.org/10.1097/MCG.0b013e31802dbdfc

[20] Abe, M., et al. (2001) Clinical Characteristics of Autoimmune Hepatitis with Histological Features of Acute Hepatitis. Hepatology Research, 21, 213-219. https://doi.org/10.1016/S1386-6346(01)00109-7

[21] EASL (2015) Clinical Practice Guidelines: Autoimmune Hepatitis. Journal of Hepatology, 63, 971-1004. https://doi.org/10.1016/j.jhep.2015.06.030

[22] Johnson, P.J. and McFarlane, I.G. (1993) Meeting Report: International Autoimmune Hepatitis Group. Hepatology, 18, 998-1005. https://doi.org/10.1002/hep.1840180435

[23] Yan, H.P., Zhang, H.P. and Chen, X.X. (2017) Meeting Report: International Autoimmune Hepatitis Group. Chinese Journal of Hepatology, 25, 63-64.

[24] Csepregi, A., et al. (2006) Budesonide Induces Complete Remission in Autoimmune 
Hepatitis. World Journal of Gastroenterology, 12, 1362-1366. https://doi.org/10.3748/wjg.v12.i9.1362

[25] Manns, M.P., et al. (2010) Budesonide Induces Remission More Effectively than Prednisone in a Controlled Trial of Patients with Autoimmune Hepatitis. Gastroenterology, 139, 1198-1206. https://doi.org/10.1053/j.gastro.2010.06.046

[26] Czaja, A.J. (2012) Diagnosis, Pathogenesis, and Treatment of Autoimmune Hepatitis after Liver Transplantation. Digestive Diseases and Sciences, 57, 2248-2266. https://doi.org/10.1007/s10620-012-2179-3

\section{Abbreviations}

- AIH: Autoimmune hepatitis

- AIHG: International auto-immune hepatitis group

- IgG: Immunoglobulin G

- ALT: Alanine aminotransferase

- AST: Aspartate aminotransferase

- ANA: Antinuclear antibodies

- SMA: Smooth muscle autoantibodies

- SLA/LP: Antibodies against soluble liver antigen/liver-pancreas

- LKM: Anti-liver/kidney microsomal antibody

- HAV: Hepatitis A virus

- HBV: Hepatitis B virus

- HCV: Hepatitis C virus

- CMV: Cytomegalovirus

- EBV: Epstein-Barr virus

- HSV: Herpes simplex virus

- ULN: Upper limit of normal

- PBC: Primary biliary cholangitis

- PSC: Primary sclerosing cholangitis 